\title{
XIAP inhibition of caspase-3 preserves its association with the Apaf-1 apoptosome and prevents CD95- and Bax-induced apoptosis
}

\author{
SB Bratton ${ }^{1}$, J Lewis ${ }^{2,3}$, M Butterworth ${ }^{1}$, CS Duckett ${ }^{2,3}$ and \\ GM Cohen*,1 \\ ${ }^{1}$ Medical Research Council Toxicology Unit, Hodgkin Building, University of \\ Leicester, P.0. Box 138, Lancaster Road, Leicester LE1 9HN, UK \\ 2 Department of Pathology, University of Michigan Medical School, Medical \\ Science Building I, Room 5315, 1301 Catherine Street, Ann Arbor, Michigan, \\ MI 48109-0602, USA \\ ${ }^{3}$ Department of Internal Medicine, University of Michigan Medical School, \\ Medical Science Building I, Room 5315, 1301 Catherine Street, Ann Arbor, \\ Michigan, MI 48109-0602, USA \\ * Corresponding author: GM Cohen, Medical Research Council Toxicology \\ Unit, Hodgkin Building, University of Leicester, P.O. Box 138, Lancaster Road, \\ Leicester LE1 9HN, UK. Tel: 44116252 5601; Fax: 44116252 5616; E-mail: \\ gmc2@le.ac.uk
}

Received 19.3.02; revised 9.4.02; accepted 9.4.02

Edited by SJ Martin

\begin{abstract}
Ligation of death receptors or formation of the Apaf-1 apoptosome results in the activation of caspases and execution of apoptosis. We recently demonstrated that Xlinked inhibitor-of-apoptosis protein (XIAP) associates with the apoptosome in vitro. By utilizing XIAP mutants, we now report that XIAP binds to the 'native' apoptosome complex via a specific interaction with the small p12 subunit of processed caspase-9. Indeed, we provide the first direct evidence that XIAP can simultaneously bind active caspases -9 and -3 within the same complex and that inhibition of caspase-3 by the Linker-BIR2 domain prevents disruption of BIR3-caspase-9 interactions. Recent studies suggest that inhibition of caspase-3 is dispensable for its anti-apoptotic effects. However, we clearly demonstrate that inhibition of caspase3 is required to inhibit CD95 (Fas/Apo-1)-mediated apoptosis, whereas inhibition of either caspase- 9 or caspase- 3 prevents Bax-induced cell death. Finally, we illustrate for the first time that XIAP mutants, which are incapable of binding to caspases-9 and -3 are completely devoid of anti-apoptotic activity. Thus, XIAP's capacity to maintain inhibition of caspase-9 within the Apaf-1 apoptosome is influenced by its ability to simultaneously inhibit active caspase-3, and depending upon the apoptotic stimulus, inhibition of caspase-9 or 3 is essential for XIAP's anti-apoptotic activity. Cell Death and Differentiation (2002) 9, 881-892. doi:10.1038/ sj.cdd.4401069
\end{abstract}

Keywords: Apaf-1 apoptosome; XIAP; caspases; Smac/DIABLO; CD95; Bax
Abbreviations: Apaf-1, apoptotic protease-activating factor 1; Smac, second mitochondrial-activator of caspases; CARD, caspase recruitment domain; DEVD.CHO, acetyl-Asp-Glu-ValAsp aldehyde; IAP, inhibitor-of-apoptosis protein; BIR, baculovirus IAP repeat; XIAP, X-linked inhibitor-of-apoptosis protein; GST, glutathione-S-transferase

\section{Introduction}

Apoptosis is a form of cell death that is essential for normal development and homeostasis of adult tissues and is characterized by specific morphological and biochemical changes. These changes ordinarily result from the action of aspartate-specific cysteine proteases, known as caspases. ${ }^{1,2}$ Caspases are synthesized as single-chain zymogens, containing an $\mathrm{N}$-terminal prodomain, as well as a large ( $\sim 20 \mathrm{kDa})$ and small $(\sim 10 \mathrm{kDa})$ subunit. Because the large subunits are flanked by aspartic acid cleavage sites, caspases can be activated by one another through unique signal transduction pathways. ${ }^{3}$ In most cases, these pathways occur within large multimeric protein complexes, which initially contain an apical caspase and its adapter molecule. ${ }^{4}$ For example, stimulation or overexpression of death receptors, such as CD95 (Fas/Apo-1) or DR5 (TRAIL-R2), results in recruitment of the adapter protein, Fas-associated death domain (FADD), to the intracellular domain of the receptor. FADD contains both a death domain (DD) and a death effector domain (DED), which allows it to simultaneously interact with homologous regions located in the receptor and caspase-8, respectively. This complex of CD95, FADD and caspase- 8 is often referred to as the death-inducing signaling complex (DISC), and its formation is required to activate caspase-8. ${ }^{5}$

Many toxicants and radiation also induce activation of caspases through a similar, yet distinct pathway. These agents often perturb mitochondria, resulting in release of cytochrome $c .^{6,7}$ Although the mechanism(s) remain unclear, pro-apoptotic Bcl-2 proteins, such as Bax and/or Bak, appear to promote disruption or permeabilization of the outer mitochondrial membrane, allowing free passage of proteins from the inner mitochondrial membrane space to the cytosol. ${ }^{8}$ Once released, cytochrome $c$ acts in concert with dATP or ATP to induce oligomerization of Apaf-1 into an 'apoptosome' complex. ${ }^{9-13}$ This complex exists structurally as a wheel-like particle with seven bent spokes that radiate from a central hub. ${ }^{14}$ Procaspase- 9 proteins are apparently recruited to the central hub, where they undergo proximity-induced, trans-catalytic processing. Remarkably, the Apaf-1 apoptosome functions as a direct allosteric regulator of caspase- 9 activity (i.e. the Apaf-1/caspase- 9 
complex is an active holoenzyme) and indeed both processed and unprocessed caspase- 9 proteins exhibit significantly greater catalytic activity when associated with the apoptosome. ${ }^{15-18}$ In some instances, stimulation of death receptors may also lead to formation of the apoptosome. Indeed, caspase- 8 may cleave and activate the proapoptotic $\mathrm{Bcl}-2$ (BH3-only) protein, Bid, which then stimulates release of cytochrome $c$ from mitochondria and consequently, formation of the apoptosome. ${ }^{19,20}$ Both caspases- 8 and -9 amplify the apoptotic signal by recruiting and activating numerous effector caspases, such as caspases-3, -6 and -7 , which are responsible for dismantling the cell by cleaving a variety of critical structural and regulatory proteins. ${ }^{2,21,22}$

Inhibitor-of-apoptosis proteins (IAPs) are evolutionarily conserved proteins that were first identified in baculoviruses. They contain tandem repeats of $\sim 70$ amino acids, termed baculovirus IAP repeat (BIR) domains, and they frequently possess a C-terminal RING zinc finger domain. ${ }^{23}$ Several IAPs have been identified in mammalian cells, and some, but not all, directly inhibit caspases, including X-linked IAP (XIAP; hILP/MIHA), ILP-2, ML-IAP (Livin), cellular inhibitor of apoptosis protein-1 (clAP-1/ HIAP-2/MIHB) and CIAP-2 (HIAP-1/MIHC). ${ }^{24-26}$ XIAP, the most potent of these caspase inhibitors, inhibits active caspase- 9 through its BIR3 domain and active caspases- 3 and -7 through its Linker-BIR2 domain. ${ }^{18,27-33}$ In addition, XIAP possesses E3 ligase activity and thus, can ubiquitinate active caspase- 3 and target it for degradation by the proteasome. ${ }^{34,35}$ The importance of caspase inhibition by XIAP appears to be substantiated, in that Smac, a 'second-mitochondrial-activator-of-caspases' (also known as DIABLO), antagonizes the interactions of XIAP with processed caspase-9, and to a lesser extent, with active caspase-3. ${ }^{18,36-40}$ Nevertheless, it remains unclear whether XIAP inhibits apoptosis solely through caspase inhibition. Indeed, XIAP is thought to participate in transforming growth factor- $\beta$ (TGF $\beta$ ), bone morphogenetic protein (BMP), c-Jun N-terminal kinase (JNK) and NF- $\kappa$ B signalling pathways. ${ }^{41-45}$ Moreover, its ability to inhibit caspase-3 is reportedly dispensable for its antiapoptotic activity. ${ }^{46}$

In a previous report, we established that XIAP associates with the Apaf-1 apoptosome, apparently through multiple interactions. ${ }^{16}$ We have now extended these studies, utilizing full-length XIAP mutants in order to specifically define the interactions of XIAP with the 'native' apoptosome complex. We find that XIAP associates with the apoptosome, primarily through an interaction involving its BIR3 domain and the small p12 subunit in processed caspase- 9 . Remarkably, maintenance of this BIR3-caspase-9 interaction is dependent upon XIAP's ability to simultaneously bind and inhibit active caspase-3 via its Linker-BIR2 domain. In contrast to previous suggestions, we also find that direct inhibition of caspase- 3 by XIAP is essential for its protection against certain forms of apoptosis. Furthermore, we clearly demonstrate for the first time that the antiapoptotic effects of XIAP can be entirely attributed to its inhibition of caspases.

\section{Results}

\section{BIR3-RING of XIAP associates with the Apaf-1 apoptosome}

Previously, we demonstrated that dATP-activation of cell lysates results in complete oligomerization of Apaf-1 into apoptosome complexes, containing active caspases-9, -3 and -7 , as well as XIAP. ${ }^{9,10,16,47}$ In the present studies, in order to determine the specific domains in XIAP responsible for its interaction with the 'native' apoptosome, we first examined two of its endogenous caspase-cleavage products, BIR1-BIR2 and BIR3-RING. ${ }^{48,49}$ Since both fragments could potentially exhibit overlapping specificities, we tested each over a range of concentrations so that their relative affinities for certain components of the apoptosome could be ascertained.

In dATP-activated 293 cell lysates, full-length XIAP and BIR3-RING bound oligomerized Apaf-1 and active caspase9 with similar affinities (Figure 1A, upper panels, lanes 2-5 and 10-13). However XIAP was significantly more potent at inhibiting the initial processing of procaspase-3 to its p20 form (Figure 1A, lower panels, lanes $2-5$ and $10-13$ ). This apparent discrepancy was probably because, unlike BIR3RING, XIAP also bound to and inhibited active caspase-3 (Figure 1A, lanes 2-5 and 10-13) and thus, prevented it from participating in the processing of additional procaspase-3. Similarly, XIAP displayed a slightly higher affinity for Smac than did BIR3-RING (Figure 1A, lanes 2-5 and 10-13), perhaps because XIAP also contains a BIR2 domain that can weakly bind to Smac (Figure 1A, lanes 69). BIR1-BIR2 did not interact with either Apaf-1 or caspase-9, but in contrast to BIR3-RING, did bind strongly to processed caspase-3 and slightly inhibited the autocatalytic conversion of its p20 subunit to its p17 form (Figure 1A, lanes 6-9 and 10-13).

Therefore, these results suggested that the BIR3-RING domain in XIAP was either directly associated with oligomerized Apaf-1 or indirectly associated with the apoptosome via an interaction with caspase-9 or Smac. In this regard, XIAP has recently been reported to interact with a reconstituted apoptosome, containing only recombinant Apaf-1 and caspase- 9 proteins, through an interaction with the small subunit of caspase- $9 .{ }^{18}$

\section{Linker-BIR2 mutant exhibits decreased affinity for caspase-9 and Apaf-1, but increased affinity for Smac/DIABLO}

The inability of BIR1-BIR2 to interact with Apaf-1 in the apoptosome was somewhat surprising to us, given that caspase-3 associates with both oligomerized Apaf-1 and XIAP. ${ }^{16}$ NMR, crystallographic and biochemical studies with truncated and mutant XIAP proteins, indicate that the BIR2 domain, including an adjacent $\mathrm{N}$-terminal linker region (Linker-BIR2), is sufficient to inhibit caspase-3. ${ }^{29,30,32,33}$ Importantly, this linker region (residues 138-156) lies across the substrate binding cleft in caspase-3, in the reverse orientation to the synthetic inhibitor DEVD.CHO, and inhibits substrate binding. ${ }^{29}$ Asp-148 appears particularly important, 
A.
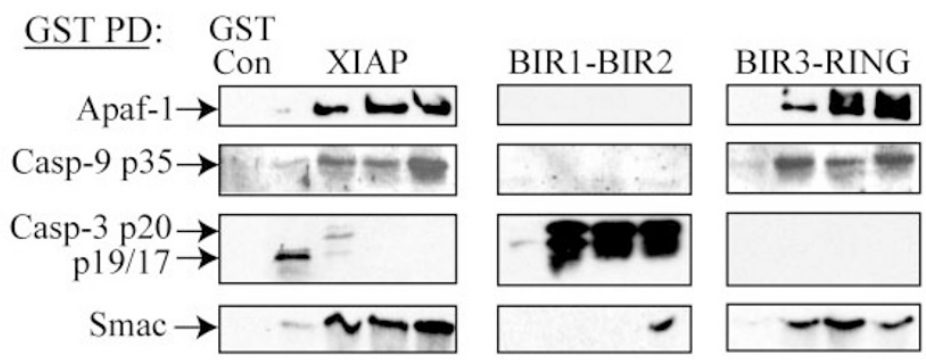

XIAP

(D148A)
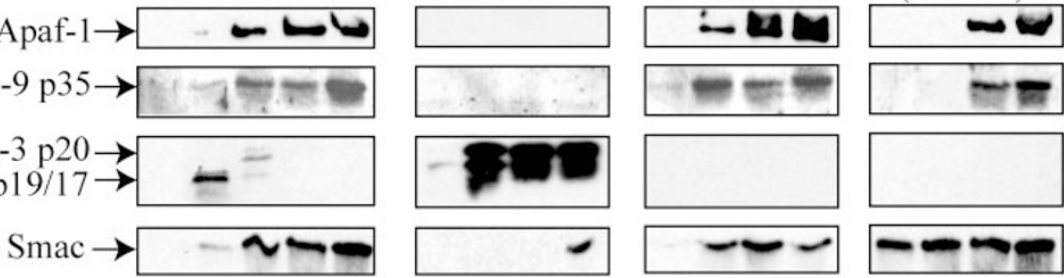

Supernatants:
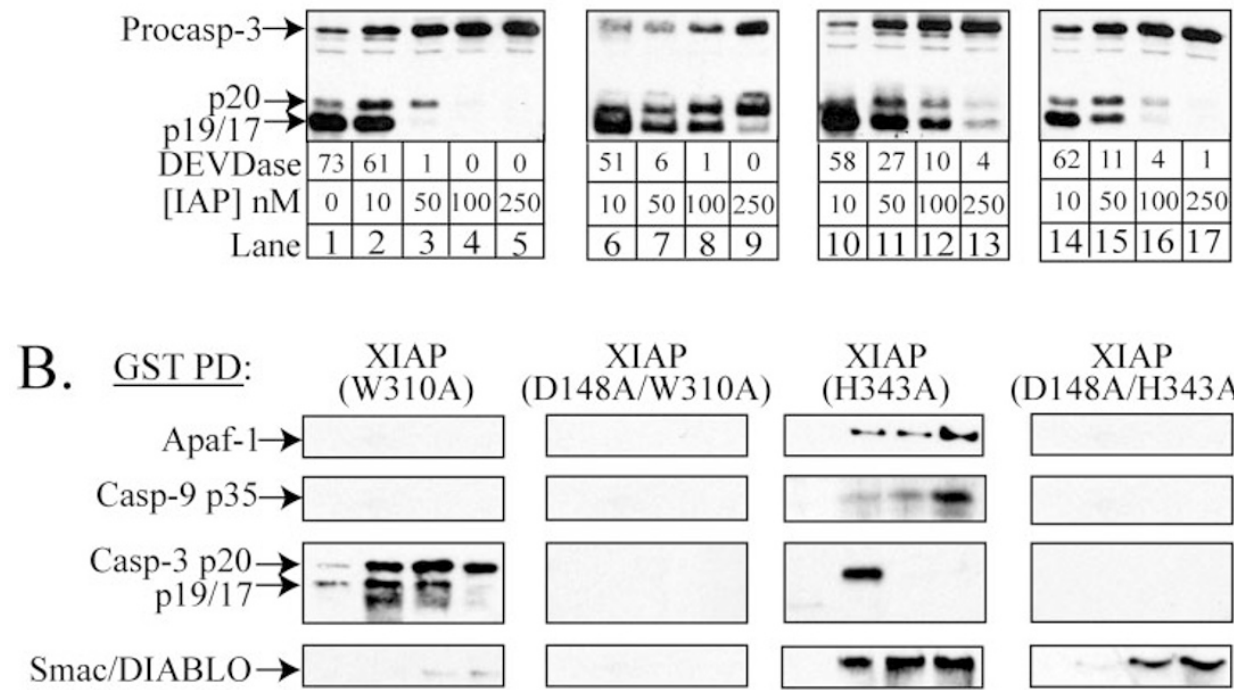

XIAP

(H343A)
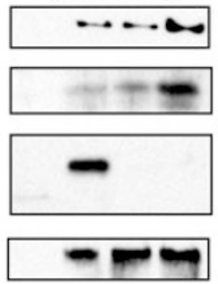

XIAP (D148A/H343A)

\section{Supernatants:}
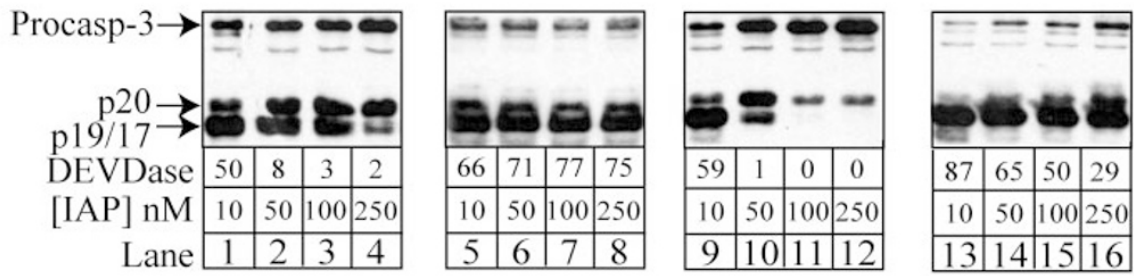

Figure 1 Molecular determinants of XIAP-Apaf-1 apoptosome interactions. (A) GST-XIAP, its cleavage products, BIR1-BIR2 and BIR3-RING, and (B) various caspase- 3 and -9 binding mutants $(10-250 \mathrm{nM})$ were added to HEK 293 cell lysates $(10 \mathrm{mg} / \mathrm{ml})$. These lysates were subsequently activated with dATP $(2 \mathrm{mM})$ and $\mathrm{MgCl}_{2}(2 \mathrm{mM})$ for $10 \mathrm{~min}$ at $37^{\circ} \mathrm{C}$. A small aliquot of each sample was immediately assayed for DEVDase activity (pmol/min), and the remaining sample was incubated with GSH-sepharose beads $(20 \mu \mathrm{l})$ for $1 \mathrm{~h}$ at $4^{\circ} \mathrm{C}$. The bead-protein complexes (GST PD) were subsequently isolated by centrifugation, washed with icecold PBS $(3 \times)$ and immunoblotted for Apaf-1, caspase-9, caspase-3 and/or Smac. A representative GST control (250 nM) is shown for comparison. Corresponding supernatants were immunoblotted for caspase- 3 , in order to assess the initial processing of procaspase-3 by caspase- 9 and the subsequent autocatalytic processing of caspase-3 from its p20/p12 to its p17/p12 form

as it forms hydrogen bonds with Arg-341, Ser-343, Trp-348 and Phe-381 in caspase-3, as well as a salt-bridge with Arg233, which helps to anchor caspase-3 next to the BIR2 domain. ${ }^{29}$ Consequently, introduction of a D148A mutation into XIAP dramatically reduces its ability to inhibit caspase$3^{30,32,34}$

In order to exclude any possible role for caspase-3 in the association of XIAP with oligomerized Apaf-1, we examined $\mathrm{XIAP}(\mathrm{D} 148 \mathrm{~A})$ in our apoptosome assay. As anticipated, full- length $\mathrm{XIAP}(\mathrm{D} 148 \mathrm{~A})(10-250 \mathrm{nM})$ did not bind active caspase-3, but did bind active caspase-9, because it contained a functional BIR3 domain (Figure 1A, upper panel, lanes 14-17). Surprisingly, however, XIAP(D148A) bound both caspase- 9 and Apaf-1 with reduced affinity compared to wild-type XIAP (Figure 1A, upper panels, lanes $14-17$ and 2-5), implying that the affinity of XIAP for caspase-9 and Apaf-1 was enhanced when XIAP was also bound to caspase-3. 
In contrast, $\mathrm{XIAP}(\mathrm{D} 148 \mathrm{~A})$ bound Smac with increased affinity compared to wild-type XIAP (Figure 1A, upper panels, lanes 14-17 and 2-5). Although no structural data are available, Smac is believed to interact with a binding groove in BIR2 that is similar to the one in BIR3 (see below). The small subunit of active caspase- 3 contains an $\mathrm{N}$-terminal 'SGVD' motif that is proposed to interact with this putative binding groove. ${ }^{29}$ Therefore, under normal circumstances, Smac may displace caspase-3 from the Linker-BIR2 region by displacing this 'SGVD' motif and perhaps, by making a conformational change in BIR2 that destabilizes the adjacent caspase-3-Linker interaction. Consequently, since the D148A mutation in XIAP dramatically reduces the overall affinity of the Linker-BIR2 for active caspase-3, Smac may have an increased advantage over caspase-3 for binding into the BIR2 pocket in XIAP(D148A).

\section{XIAP mutants that fail to bind caspase-9 do not associate with the Apaf-1 apoptosome}

As already noted, the ability of BIR3-RING to bind Apaf-1 in dATP-activated lysates, suggested that XIAP might bind the apoptosome through an indirect association with either caspase-9 or Smac (Figure 1A). Previous analysis of the truncated BIR3 domain revealed the existence of a putative caspase-9 binding site, which appeared to partially overlap with a well-defined Smac binding pocket. ${ }^{18,31,50-52}$ Indeed, W310A and E314S mutations in BIR3 inhibit its binding to caspase-9 and Smac, whereas mutation of His-343 to alanine reportedly affects only its interaction with caspase- $9 .{ }^{31,50}$ Structurally, Glu-314 is located in a negatively charged patch, and His-343 and Trp-310 are located nearby, on the edge of a basic patch that forms a shallow pocket on the surface of the protein. ${ }^{31}$ An 'ATPF' motif located on the N-terminus of the small (p12) subunit of caspase-9, and a similar 'AVPI' motif on the N-terminus of mature Smac, appear to fit into this pocket and are absolutely required for their interactions with BIR3. ${ }^{18,50-52}$

In our apoptosome model, we found that full-length XIAP(W310A) did not bind to oligomerized Apaf-1 or caspase-9 (Figure 1B, lanes 1-4). However, since it retained a normal Linker-BIR2 domain, it behaved similarly to BIR1-BIR2 in that it bound weakly to Smac, but strongly to processed caspase- 3 and thus, partially inhibited autocatalytic conversion of its p20 subunit to its p17 form (Figure 1B, lanes 1-4; Figure 1A, lanes 6-9). We next prepared XIAP(D148A/W310A) and found that it was totally incapable of binding to Apaf-1, caspase-9 or caspase-3 (Figure 1B, upper panels, lanes 5-8). Furthermore, this double mutant did not inhibit the activation, autocatalytic processing or DEVDase activity of caspase-3 at any concentration (Figure 1B, lower panel, lanes 5-8). In fact, even though this protein contained a D148A mutation, and might be expected to exhibit increased binding to Smac, the excessive amount of active caspase-3 probably precluded any chance of binding to Smac.

We previously reported that XIAP can interact with the apoptosome in caspase-9-depleted THP.1 lysates, ${ }^{16}$ indicating that a factor, other than caspase- 9 , might provide a bridge between XIAP and Apaf-1. Therefore, since XIAP(W310A) dramatically disrupted interactions with both caspase-9 and Smac, we prepared $\operatorname{XIAP}(\mathrm{H} 343 \mathrm{~A})$ in order to determine if Smac contributed to XIAP-Apaf-1 interactions. Surprisingly, we found that in addition to binding Smac, XIAP(H343A) could bind caspase-9 and Apaf-1, albeit to a lesser extent than wild-type XIAP (Figure 1B, lanes 9-12; Figure 1A, lanes 2-5). Moreover, the H343A mutant inhibited caspase-9-mediated processing of procaspase-3, but was not as potent as wild-type XIAP (Figure $1 \mathrm{~B}$, lower panel, lanes 9-12; Figure 1A, lower panel, lanes $2-5)$. These results were in conflict with those previously reported by Fesik and colleagues for the same mutant. ${ }^{31}$ Importantly, however, in their study, the H343A mutant was introduced into a truncated BIR3 fragment (residues 252356) of XIAP. ${ }^{31}$ Therefore, we prepared BIR3-RING $(\mathrm{H} 343 \mathrm{~A})$ and found that it was indeed less effective at binding caspase- 9 compared to full-length $\operatorname{XIAP}(\mathrm{H} 343 \mathrm{~A})$, but nonetheless still bound and inhibited caspase- 9 at higher concentrations (>100 nM; data not shown). These results underscore the importance of testing the functionality of any mutation in full-length proteins. In any event, when we generated $\mathrm{XIAP}(\mathrm{D} 148 \mathrm{~A} / \mathrm{H} 343 \mathrm{~A})$, we found that it could no longer bind Apaf-1, caspase- 9 or caspase-3, but it retained an ability to bind Smac (Figure 1B, lanes 13-16).

Therefore, XIAP specifically associates with the Apaf-1 apoptosome via an interaction with caspase-9, at least in HEK 293 cell lysates, since XIAP mutants that failed to bind processed caspase-9, even when they maintained an association with Smac, were unable to interact with the native apoptosome. In monocytic THP.1 cells, it remains unclear what additional factor(s) may be present that can link XIAP with the apoptosome. Equally important, however, were the observed differences in the binding of XIAP (H343A) and XIAP(D148A/H343A) to caspase-9 (Figure $1 \mathrm{~B}$, upper panels, lanes $9-12$ and 13-16). These data once again suggested that the association of caspase- 3 with the Linker-BIR2 influenced the binding of caspase- 9 to the BIR3 domain.

\section{Inhibition of caspase-3 activity by the Linker-BIR2 of XIAP prevents processing of the caspase-9 p12 subunit and disruption of caspase-9-BIR3 interactions}

The observed differences in caspase- 9 binding between XIAP and $\mathrm{XIAP}(\mathrm{D} 148 \mathrm{~A})$ (Figure 1A), as well as XIAP(H343A) and $\mathrm{XIAP}(\mathrm{D} 148 \mathrm{~A} / \mathrm{H} 343 \mathrm{~A}$ ) (Figure 1B), suggested that XIAP might exhibit cooperative binding between its Linker-BIR2 and BIR3 domains. However, since all of the D148A mutants were incapable of inhibiting active caspase-3, it was also possible that caspase-3 might process procaspase-9 to its $\mathrm{p37/p10}$ form, which cannot be inhibited by $\mathrm{XIAP}^{18}$ or alternatively, might disturb existing caspase-9 (p35/p12)-BIR3 interactions by converting the $\mathrm{p} 12$ to a $\mathrm{p} 10$ subunit. To address these two possibilities, we examined XIAP and the D148A, H343A and D148A/H343A mutants in the presence and absence of DEVD.CHO. This reversible inhibitor selectively binds the active site of caspase- 3 and inhibits its processing of substrates, such as caspase- $9 .{ }^{53}$ However, in the presence 
of XIAP, DEVD.CHO also competes with the Linker-BIR2 domain for inhibition of caspase-3. ${ }^{16,30}$ Therefore, we reasoned that if caspase- 3 binding cooperatively increased association of active caspase- 9 with the BIR3 domain, the presence of DEVD.CHO should decrease caspase-9 binding to wild-type XIAP and the H343A mutant, but should have no effect on D148A mutants, since these proteins are already incapable of binding caspase-3. In contrast, if caspase-3 processed procaspase- 9 to a p37/p10 heterotetramer, or caspase-9 (p35/p12) to a p35/p10 heterotetramer, DEVD. $\mathrm{CHO}$ should inhibit this cleavage and in turn, promote binding of caspase-9 to the BIR3 domain in XIAP(D148A) and XIAP(D148A/H343A) mutants.

DEVD.CHO, at the concentration utilized in these experiments, inhibited the activity of caspase-3, including its autocatalytic processing from a p20 to p17 subunit, but did not inhibit the activation of caspase- 9 or its ability to initially process procaspase-3 (Figure 2A, B, lower panels). $\mathrm{XIAP}$, in the presence of DEVD.CHO, no longer associated with processed caspase- 3 and appeared to bind caspase- 9 and Apaf-1 with slightly decreased affinity (Figure 2A, upper panels, lanes 2-6 and 7-11). These data supported the suggestion that some subtle cooperative binding might indeed exist. However, DEVD.CHO increased binding of caspase-9 and Apaf-1 to XIAP(D148A), XIAP(H343A) and $\mathrm{XIAP}(\mathrm{D} 148 \mathrm{~A} / \mathrm{H} 343 \mathrm{~A})$ (Figure 2A, B, upper panels). The apparent affinity of $\mathrm{XIAP}(\mathrm{D} 148 \mathrm{~A})$ and $\mathrm{XIAP}(\mathrm{H} 343 \mathrm{~A})$ for caspase- 9 and Apaf-1 increased, such that binding was readily observed at $50 \mathrm{nM}$, compared to $100 \mathrm{nM}$ in the absence of DEVD.CHO (Figure 2A, lanes $14-16$ and 1921; Figure $2 \mathrm{~B}$, lanes $5-6$ and $9-11)$. Furthermore, in the case of $\mathrm{XIAP}(\mathrm{D} 148 \mathrm{~A} / \mathrm{H} 343 \mathrm{~A})(250 \mathrm{nM})$, this mutant could only bind caspase- 9 and Apaf-1 in the presence of DEVD.CHO (Figure 2B, lanes 16 and 21).

Since the D148A mutants could not bind active caspase3 , it appeared likely that DEVD.CHO was substituting for the absence of a Linker-BIR2 domain. However, XIAP (H343A) contained a functional Linker-BIR2 domain, which should inhibit caspase-3 and yet, the presence of DEVD.CHO still improved its binding to caspase- 9 and Apaf-1 (Figure 2B, lanes 5-6 and 9-11). It is important to note, however, that the H343A mutant is somewhat deficient in its ability to bind caspase- 9 and inhibit the activation of caspase-3, compared to wild-type XIAP. Consequently, there was more processed caspase-3 present in the incubations containing $\operatorname{XIAP}(\mathrm{H} 343 \mathrm{~A})$, than with XIAP (Figure 2B, lower panel, lanes 2-4; Figure 2A, lower panel, lanes 2-3). Indeed, after careful examination, we noticed an important trend in the data. In every incubation, where large amounts of processed caspase-3 were present, the XIAP mutants exhibited significantly less binding to caspase-9 and Apaf-1 (Figure 2A, lanes 12-14; Figure 2B, lanes $1-4$ and 12-16). However, when DEVD.CHO was present, binding to caspase-9 and Apaf1 was enhanced and observed at the next lowest concentration (Figure 2A, lane 19; Figure 2B, lanes 9 and 21). Consequently, we immunoblotted all supernatants with a specific antibody that recognizes only the p12 subunit, and not the p10 subunit of caspase-9. Generally, we found that the presence of a p12 subunit directly correlated with binding of XIAP mutants to caspase-9 (Figure 2A, B, lower panels). Therefore, in those incubations where active caspase-3 was present, it processed the p12 subunit of caspase- 9 to a p10 subunit, and the resulting caspase- 9 (p35/p10) heterotetramer was no longer capable of binding to XIAP

However, closer inspection of the data did reveal some subtle inconsistencies. If caspase-9 (p35/p12) associates with XIAP through p12-BIR3 interactions, which can be disturbed by caspase-3 activity, then why did wild-type XIAP (10 and $25 \mathrm{nM}$ ) bind caspase- 9 and Apaf-1, even in the absence of DEVD.CHO, when the p12 subunit was not observed in supernatants (Figure 2A, lanes 2-3)? Conversely, if DEVD.CHO fully substitutes for the absence of a functional Linker-BIR2 and prevents caspase-3 from processing the $p 12$ subunit of caspase- 9 , then why was the binding observed with $\operatorname{XIAP}(D 148 A)$ and $\operatorname{XIAP(D148A/~}$ $\mathrm{H} 343 \mathrm{~A})$, in the presence of DEVD.CHO, not identical to wild-type XIAP and XIAP(H343A), respectively (Figure 2A, $B$, lanes $17-21$ compared to lanes $2-6$ or $7-11)$ ? We propose that the answer to both questions involves the relative capacities of the Linker-BIR2 and DEVD.CHO to protect caspase-9 (p35/p12)-BIR3 interactions. Indeed, the data support the hypothesis that by having a caspase-3 binding motif (Linker-BIR2) directly juxtaposed to the caspase- 9 binding site (BIR3), XIAP can specifically protect the p12 subunit of bound caspase-9, far more effectively than freely diffusible DEVD.CHO (Figure 5, model 3 versus 4). Furthermore, even at low concentrations of wild-type XIAP, a functional Linker-BIR2 domain can preserve established caspase-9-BIR3 interactions, but cannot inhibit all caspase- 3 activity outside the complex (Figure $2 \mathrm{~A}$, lanes $2-3$ ) and thus, cannot prevent processing of unbound caspase-9.

\section{XIAP simultaneously associates with caspases-9 and -3}

In order for the Linker-BIR2 to inhibit caspase-3 and consequently, preserve caspase-9-BIR3 interactions, XIAP would have to be capable of binding both caspases- 9 and -3 within the same complex. In our previous report, we indicated that XIAP interacts with both caspases-9 and -3; however, immunoprecipitation of caspase- 9 did not reveal an interaction with caspase- 3 or vice versa, ${ }^{16}$ suggesting that all three proteins were not present within the same complex. Nevertheless, as these native proteins were untagged, we could not rule out the possibility that our antibodies disrupted caspase9/XIAP/caspase-3 complexes.

Therefore, in the present studies, we expressed recombinant T7-tagged caspase-9 (p35/p12) and caspase-3 (p17/p12) in bacteria and purified the enzymes to homogeneity. We then incubated them in various combinations with GST-tagged XIAP, and immunoprecipitated complexes with anti-T7 antibodies. In the absence of caspase- 9 , there were no nonspecific interactions between the antibody-coated beads and XIAP or caspase-3 (Figure $3 A$, lane 3). T7-tagged caspase-9, however, did interact strongly with XIAP (Figure $3 \mathrm{~A}$, lane 2). This indicates that processed caspase-9 (p35/p12) can interact with XIAP, 
A.

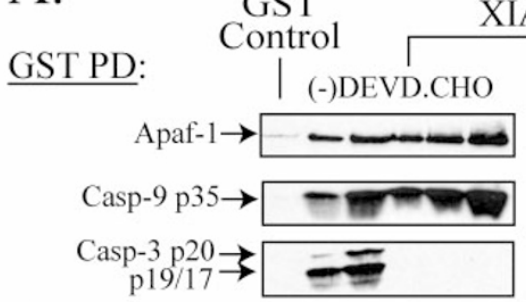

Supernatants:

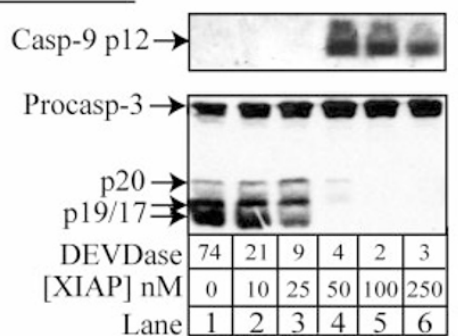

GST ntrol

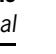

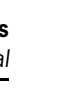


A.

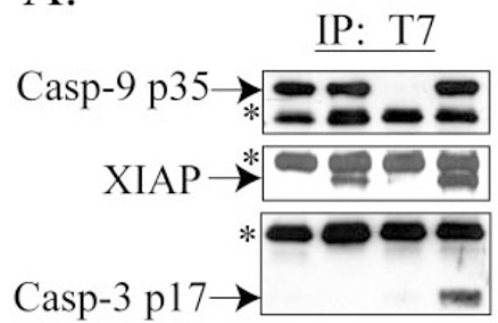

Supernatants

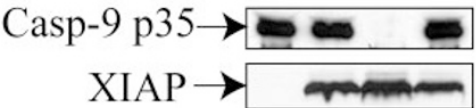

\begin{tabular}{r|l|l|l|l|}
\cline { 3 - 5 } Casp-3 p17 & \multicolumn{4}{|c|}{} \\
T7-Casp-9 & + & + & - & + \\
\cline { 3 - 6 } XIAP & - & + & + & + \\
Casp-3 & + & - & + & + \\
\cline { 3 - 6 } Lane & 1 & 2 & 3 & 4 \\
\hline
\end{tabular}
B.

GST-XIAP PD:

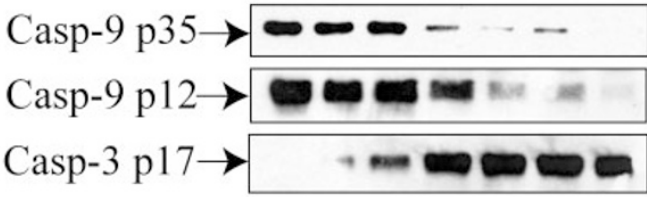

Supernatants:

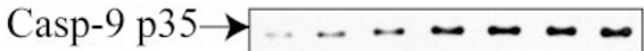

Casp-9 p12 $\rightarrow--\infty-$

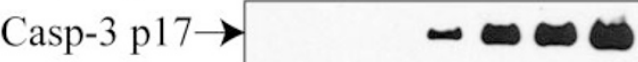

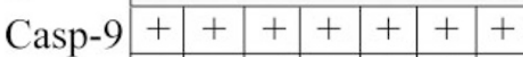

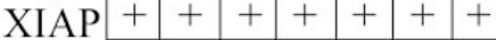

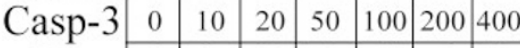

Figure 3 XIAP simultaneously associates with caspases-9 and -3, but BIR3-p12 caspase-9 interactions are susceptible to 'clipping' by excessive caspase-3. (A) Purified GST-XIAP (60 nM), T7-tagged caspase-9 (p35/p12) (200 nM) and active caspase-3 (p17/p12) (200 nM) were incubated together, as indicated, for 15 min at $25^{\circ} \mathrm{C}$. Beads coated with anti-T7 antibodies were subsequently added to each sample and incubated for an additional 45 min at $25^{\circ} \mathrm{C}$. XIAP/caspase-9/caspase-3 complexes were then isolated by centrifugation, washed and analyzed by SDS - PAGE/Western blotting. The asterisk (*) denotes cross reactivity with the heavy or light chains of the T7 antibody. (B) Similarly, GST-XIAP (60 nM) was incubated with excess caspase-9 (p35/p12) for 30 min at $4^{\circ} \mathrm{C}$. The GST-XIAP/caspase-9 complexes were then isolated with GSH-sepharose beads, washed $(3 \times)$ with ice-cold PBS, and subsequently incubated with increasing concentrations $(0-$ $400 \mathrm{nM}$ ) of active caspase- 3 for $30 \mathrm{~min}$ at $4{ }^{\circ} \mathrm{C}$. The resulting complexes and supernatants were obtained by centrifugation and immunoblotted for the p35 and p12 subunits of caspase- 9 , as well as caspase-3

contained a small p12 subunit (Figure 3B, upper panel, lanes 1-4). Indeed, at modest concentrations, when XIAP bound a significant portion of the available caspase-3, caspase-9 was also observed in the complex (Figure 3B, lanes 3-4). However, at higher concentrations, much of caspase-3 was not bound or inhibited by XIAP (Figure 3B, lanes 5-7). Consequently, caspase-3 processed the p12 subunit of caspase- 9 , causing caspase- 9 to dissociate from $\mathrm{XIAP}$ and relocalize to the supernatant fraction (Figure 3B, lanes 5-7). Thus, as was the case with native XIAPapoptosome complexes, unrestrained activity of caspase-3 negatively affected the binding of XIAP to caspase- 9 .

\section{XIAP inhibits CD95- and Bax-induced apoptosis exclusively through inhibition of caspases}

As already noted, there has been significant debate as to the relative importance of caspase inhibition (in particular caspase-3 inhibition) to the antiapoptotic effects of XIAP. Given that we had characterized a number of full-length XIAP mutants, at least in regard to their association with caspases, Smac and the apoptosome, we subsequently examined the antiapoptotic effects of these mutants in vivo. In order to assess their effects against death receptor- and stress (or mitochondrial)-induced apoptosis, we ectopically expressed XIAP (or one of its mutants) along with CD95 or Bax. Expression of CD95 alone led to a significant decrease in cell viability, which was completely alleviated by coexpression of wild-type XIAP (Figure 4A). In addition, when cells were cotransfected with XIAP(W310A) or XIAP(H343A), two mutants which displayed complete or partial defects in caspase-9 binding (Figure 1B), CD95-mediated apoptosis was still largely inhibited (Figure 4A). However, when cells were cotransfected with XIAP(D148A), XIAP(D148A/W310A) or $\mathrm{XIAP}(\mathrm{D} 148 \mathrm{~A} / \mathrm{H} 343 \mathrm{~A})$, there was no significant protection against cell death (Figure 4A). Indeed, only XIAP mutants, that lacked the capacity to bind and inhibit caspase-3, exhibited a loss in antiapoptotic function. Therefore, in these so-called Type I cells, in which mitochondria play little if any role in death receptor-mediated cell death, ${ }^{54}$ inhibition of caspase- 3 by XIAP was sufficient to fully protect against CD95-mediated apoptosis (Figure 4A). Importantly, we and others have also demonstrated that during CD95 or DR5induced apoptosis in Type II cells, permeabilization of mitochondria is required to release Smac/DIABLO, which subsequently antagonizes XIAP-mediated inhibition of caspase-3. ${ }^{40,55,56}$ Thus, despite previous reports, ${ }^{46}$ inhibition of caspase- 3 appears to be a general mechanism by which XIAP inhibits death receptor-induced apoptosis.

Cell death induced by Bax overexpression was somewhat more complicated. Wild-type XIAP, as well as the D148A, W310A and H343A mutants significantly protected cells against Bax-mediated apoptosis, indicating that an ability to inhibit either caspase-3 or caspase- 9 was sufficient to inhibit cell death (Figure 4B). Surprisingly, however, $\mathrm{XIAP}(\mathrm{D} 148 \mathrm{~A} / \mathrm{H} 343 \mathrm{~A})$ also inhibited Bax-induced 

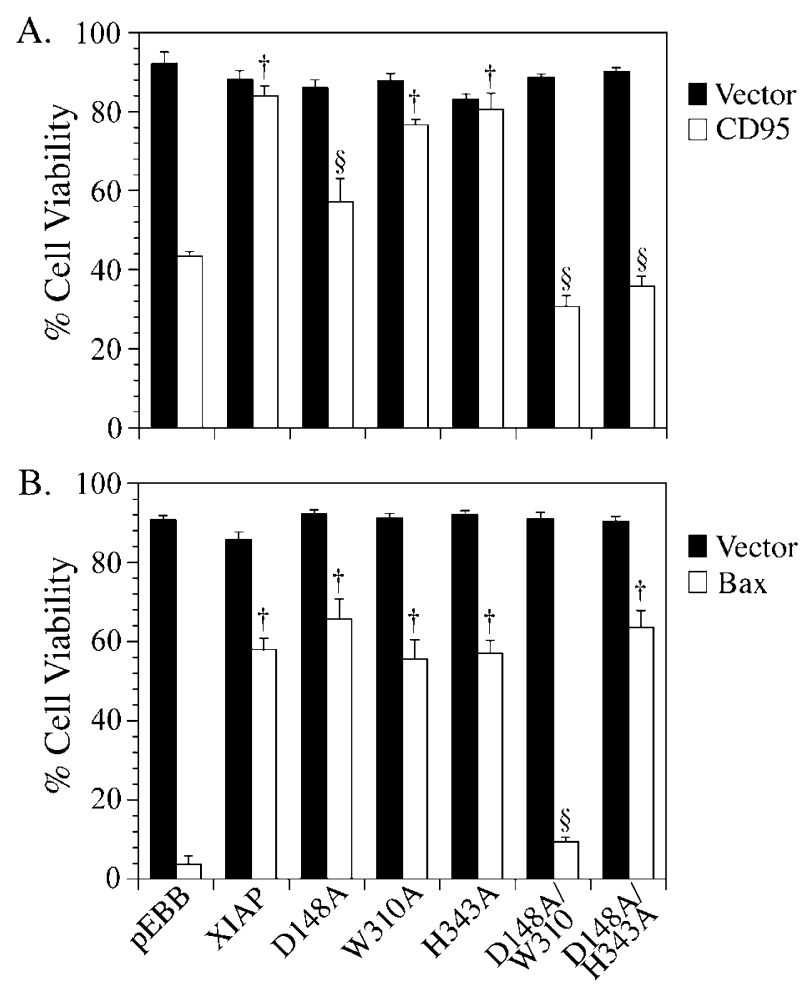

Figure 4 Inhibition of CD95 and Bax-induced apoptosis by XIAP mutants. HEK 293 cells were co-transfected for $16-24 \mathrm{~h}$ with plasmids encoding (A) CD95 $(2 \mu \mathrm{g} / \mathrm{well})$ or (B) Bax $(0.5 \mu \mathrm{g} / \mathrm{well})$, along with the transfection marker, GFP $(0.5 \mu \mathrm{g} / \mathrm{well})$, and XIAP or one of its caspase-binding mutants $(2 \mu \mathrm{g} / \mathrm{well})$. The expression levels of wild-type and mutant XIAP proteins were essentially the same (data not shown). The percentage of GFP-positive apoptotic cells was determined by cell morphology. Data shown are representative of at least three independent experiments. $\uparrow P<0.01, \mathrm{XIAP}$ and XIAP mutants that significantly protected against coexpression of $\mathrm{CD} 95$ or $\mathrm{Bax} ; \S P<0.01, \mathrm{XIAP}$ mutants that were significantly different from wild-type XIAP

cell death, to the same extent as wild-type XIAP (Figure 4B). There were at least two potential explanations for the latter result. Firstly, in vitro dATP-activation of cell lysates normally leads to robust formation of the apoptosome and activation of caspases. The significant differences observed in caspase- 9 binding, between the $\mathrm{H} 343 \mathrm{~A}$ and $\mathrm{D} 148 \mathrm{~A} /$ H343A mutants (Figure 1B, lanes $9-12$ and 13-16), suggest that caspase-9-BIR3 interactions in XIAP(D148A/ H343A) are particularly susceptible to the effects of caspase-3. Since the levels of active caspase-3 are likely to be lower in intact cells initially undergoing apoptosis, it is possible that XIAP(D148A/H343A) retained a reduced, but sufficient capacity to inhibit caspase- 9 and cell death at this time point. Intriguingly, however, XIAP(D148A/H343A) did maintain a significant interaction with Smac in dATPactivated 293 cell lysates (Figure 1B, lanes 9-12 and 13-16). Therefore, it is possible that this mutant could serve as a 'sink' for Smac, preventing this IAP antagonist from interacting with endogenous wild-type XIAP, which might then bind and inhibit active caspases-9 and -3. In any event, XIAP(D148A/ W310A), which was completely devoid of any binding to caspases or Smac in vitro, was also incapable of preventing Bax-induced cell death (Figure 4B).
Thus, XIAP can inhibit Bax-induced apoptosis (and likely apoptosis induced by any agent that stimulates mitochondrial release of cytochrome $c$ ), so long as it possesses an ability to inhibit either caspase- 9 or caspase-3.

\section{Discussion}

In the present study, we initially sought to extend upon our previous work, ${ }^{16}$ and in doing so, define the specific interactions required for the association of XIAP with the native apoptosome complex. In the interim, Alnemri and colleagues reported that XIAP could associate with a recombinant Apaf-1/caspase- 9 apoptosome in vitro by specifically interacting with an 'ATPF' motif located on the $\mathrm{N}$-terminus of the small p12 subunit of caspase- $9 .{ }^{18}$ In order to determine all of the possible interactions that XIAP might employ to associate with the apoptosome, we chose instead another approach, whereby we utilized 'native' apoptosome complexes, in combination with selective mutants of XIAP. By doing so, we hoped to specifically determine the roles of caspases-9, -3, Smac/DIABLO and any other protein (Aven, Hsp70, Hsp90) in mediating the association of XIAP with the apoptosome. We found that, in fact, XIAP mutants that lacked the capacity to bind caspase- 9 were similarly incapable of associating with the apoptosome. However, when assessing the importance of caspase-3 in mediating such an interaction, we also identified that a unique and novel interplay occurs between the Linker-BIR2 and BIR3 domains in XIAP. Indeed, we show for the first time that XIAP can simultaneously bind both active caspases- 9 and -3 , and that in a native apoptosome complex, inhibition of caspase-3 prevents additional processing of the small p12 subunit in caspase- 9 and consequently disruption of XIAP-apoptosome interactions (Figure 5).

Interestingly, if one were given the task of designing an efficient inhibitor of a proteolytic cascade, it would seem rational to target the most upstream or apical protease, in order to prevent amplification of the pathway. In the case of XIAP, however, this bifunctional inhibitor inhibits the downstream protease, caspase-3, with greater potency than the apical protease, caspase-9. Indeed, very low concentrations of XIAP are sufficient to inhibit the DEVDase activity of caspase-3, but higher concentrations are required to inhibit caspase-9-mediated activation of caspase-3. There are perhaps two potential explanations for such an evolutionary design. Firstly, as already discussed, inhibition of caspase-3 activity is crucial for maintenance of XIAP-caspase- 9 interactions and consequently, inhibition of stress-induced apoptosis. Secondly, in many tissues, death receptor stimulation can directly activate caspases- 8 and -3 , and induce cell death without mitochondrial involvement. Indeed, in contrast to previous suggestions, we show here that XIAP can inhibit apoptosis exclusively through inhibition of caspase-3, when apoptosis is induced by a death receptor, such as CD95. Therefore, XIAP may inhibit caspase-3 more potently than caspase-9, because this effector caspase is central to both death receptor- and stress-induced apoptosis. Finally, using a specific full length mutant (D148A/W310A) of XIAP, we clearly demonstrate for the first time that XIAP can only 


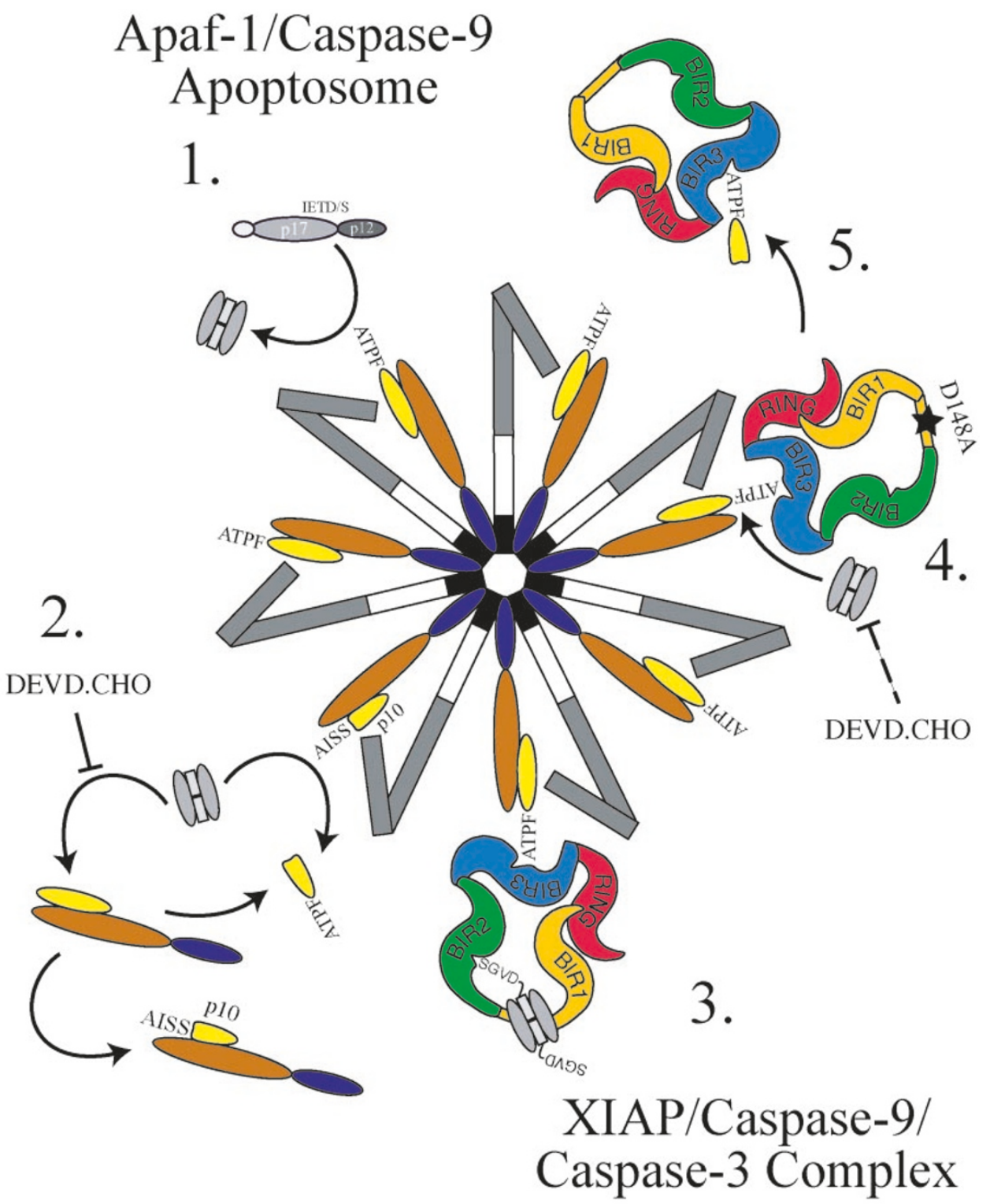

Figure 5 Linker-BIR2 and BIR3 domains cooperate for maintenance of XIAP-Apaf-1 apoptosome complexes. Release of cytochrome $c$ from mitochondria frequently occurs following an apoptotic stress. Cytochrome $c$, in cooperation with dATP/ATP, induces oligomerization of Apaf-1 into an apoptosome complex. The three-dimensional structure of this complex has recently been determined at low resolution ( $27 \AA$ ) by electron cryomicroscopy, and it indicates that the apoptosome is a wheel-like structure with seven bent spokes that radiate from a concave central hub. ${ }^{14}$ Procaspase-9 binds to the hub via CARD - CARD interactions and subsequently undergoes autocatalytic processing at D315 to produce a p35/p12 heterotetramer. (1) Caspase- 9 can then process procaspase-3 at an IETD/S motif to form a p20/p12 heterotetramer, which subsequently undergoes autocatalytic processing to form the fully mature p17/p12 heterotetramer. (2) Afterwards, active caspase- 3 can feed-back on caspase- 9 and process it at D330 to produce a p35/p10 heterotetramer, a step which can be effectively inhibited by DEVD.CHO. The presence of a p10 versus a p12 subunit in processed caspase-9, however, determines whether the enzyme can be inhibited by XIAP. (3) Indeed, the BIR3 domain in XIAP only interacts with an ATPF motif located on the N-terminus of the p12 subunit of caspase-9. Therefore, in order to protect this interaction, XIAP, via its Linker-BIR2 domain, binds tightly to any active caspase-3 which comes into close proximity of the caspase-9-BIR3 interaction. (4) However, if XIAP contains a mutated Linker-BIR2 that cannot inhibit caspase-3, e.g. D148A mutants (or if the amount of active caspase-3 exceeds the inhibitory capacity of XIAP), caspase-3 may then cleave the 12 subunit of XIAP-bound caspase- 9 and convert it to a p10 subunit. DEVD.CHO can partially inhibit this cleavage step, but not as potently as the Linker-BIR2 domain. (5) In any event, cleavage of the p12 subunit of caspase-9 to a p10 subunit results in dissociation of XIAP from the apoptosome and leaves behind a form of caspase-9 (p35/p10) which is catalytically active, but no longer inhibitible by XIAP

inhibit CD95 or Bax-induced apoptosis, provided it maintains an ability to inhibit caspases.

Of course, both wild-type and mutant XIAP proteins were present at supraphysiological concentrations in our trans- fection experiments in order to determine their dominant effects, and questions have been raised as to the relative importance of endogenous XIAP in regulating apoptosis. Indeed, in stark contrast to the global early embryonic cell 
death observed in diap-1-1- flies, $x i a p^{-/-}$mice appear to develop normally and do not exhibit any obvious changes in caspase activation or apoptosis. ${ }^{57,58}$ However in Drosophila, DIAP-1 appears to be the only significant caspase inhibitor, whereas in mammalian cells, in addition to XIAP, at least four IAPs (ILP-2, ML-IAP, CIAP-1 and clAP-2) are also capable of inhibiting caspases. In fact, deletion of XIAP in mice, led to a compensatory upregulation of both cIAP-1 and clAP-2, and may explain why $x i a p^{-1-}$ mice do not exhibit a more profound phenotype. It remains to be seen if these additional IAPs associate with and inhibit the Apaf-1 apoptosome, in a manner similar to XIAP, and whether IAPs in general are as important in mammals as they appear to be in flies.

\section{Materials and Methods}

\section{cDNA cloning and expression of recombinant proteins}

BIR1-BIR2 (residues 1-242) and BIR3-RING (residues 243-497) truncations were generated by standard PCR and cloned into pGEX4T-1 (Amersham Pharmacia Biotech, UK), in-frame with the Nterminal GST tag. XIAP mutants were prepared using the QuikChange site-directed mutagenesis kit (Stratagene, La Jolla, CA, USA). All wild-type, truncated and mutant XIAP proteins, as well as recombinant caspases- 9 and -3 , were expressed in $E$. coli strain BL21(DE3) (Novagen, Madison, WI, USA), isolated on GSH- or $\mathrm{Ni}^{2+}$ sepharose beads, and further purified by standard anion-exchange chromatography. Since purified recombinant XIAP and its mutants are susceptible to degradation, as previously reported, ${ }^{59}$ we performed a final examination of all proteins by coomassie blue staining and SDS PAGE/Western blotting to ensure they remained intact (data not shown).

\section{Preparation and activation of cell lysates}

HEK 293 cells were grown in DMEM, supplemented with $10 \%$ heatinactivated $\mathrm{FBS}$ in $5 \% \mathrm{CO}_{2}$ at $37^{\circ} \mathrm{C}$. Lysates $(100000 \times \mathrm{g})$ were prepared, as previously described, ${ }^{9}$ except that cells were lysed with 40 strokes through a 23 gauge needle. In vitro activation of caspases was initiated by incubating lysates $(10 \mathrm{mg} / \mathrm{ml})$ for $10 \mathrm{~min}$ with $2 \mathrm{mM}$ $\mathrm{dATP}$ and $2 \mathrm{mM} \mathrm{MgCl} 2$ at $37^{\circ} \mathrm{C}$. Exogenous cytochrome $c$ was not required for activation, as it was released during lysate preparation. The activity of effector caspases was determined by monitoring DEVDase activity. ${ }^{9}$

\section{Analysis of XIAP mutants and the apoptosome}

Various concentrations of purified recombinant GST-XIAP, GST-BIR1BIR2, GST-BIR3-RING and all mutants were added to lysates prior to dATP-activation. Protein complexes were obtained from control and dATP-activated lysates using GSH-sepharose beads (Amersham Pharmacia Biotech, UK) and were immunoblotted using antibodies to Apaf-1 (R\&D Systems, Minneapolis, MN, USA), caspase-9 (kindly provided by Dr D Green), caspase-3 (generously provided by Dr D Nicholson) and Smac. The anti-Smac antibody was raised in our laboratory against purified $\operatorname{Smac} \beta$ protein. ${ }^{60}$ In some cases, antibodies specific for the large subunit (p35) or small subunit (p12) of caspase-9 were also utilized (Biosource International, Camarillo, CA, USA; Cell Signaling Technology, Beverly, MA, USA).

\section{Immunoprecipitation of purified caspase-9/XIAP/ caspase-3 complexes}

Prior to the experiments, protein $\mathrm{G}$ sepharose beads were incubated for $45 \mathrm{~min}$ at $25^{\circ} \mathrm{C}$ in a solution of assay buffer (100 mM HEPES, $0.1 \%$ CHAPS, $10 \mathrm{mM} \mathrm{DTT}, 10 \%$ sucrose, $\mathrm{pH} 7.0$ ), containing 3\% BSA and anti-T7 antibodies (1: 100, Novagen). Purified GST-XIAP (60 nM), T7tagged caspase-9 (p35/p12) (200 nM) and active caspase-3 (p17/p12) $(200 \mathrm{nM})$ were then incubated together in various combinations for $15 \mathrm{~min}$ at $25^{\circ} \mathrm{C}$. Afterwards, $20 \mu \mathrm{L}$ of antibody-coated beads were washed $(3 \times)$, added to each sample and incubated for an additional $45 \mathrm{~min}$ at $25^{\circ} \mathrm{C}$. Protein complexes and corresponding supernatants were then isolated by centrifugation and analyzed by SDS-PAGE/ western blotting.

\section{Transfections and cell viability assays}

HEK 293 cells were transfected using calcium phosphate, as previously described. ${ }^{61}$ XIAP mutants were generated using the Quik-Change site-directed mutagenesis kit (Stratagene, La Jolla, CA, USA). HEK 293 cells were transfected for 16-24 h with GFP (eGFP$\mathrm{N} 1$; Clontech, Palo Alto, CA, USA) and XIAP (pEBB-XIAP wild-type or mutant) together with plasmids for CD95 (kindly provided by $\mathrm{R}$ Siegel) or Bax (generously provided by S Korsmeyer). The expression levels of wild-type and mutant XIAP proteins were essentially the same, as determined by Western blotting (data not shown). GFP-positive apoptotic cells were determined by cell morphology.

\section{Statistical analyses}

When appropriate, statistical significance was determined using a completely randomized, one-way Analysis of Variance (ANOVA), and multiple comparisons were made using Scheffé's post hoc analysis.

\section{Acknowledgements}

Antibodies to caspases-9 and -3 were generously provided by Drs $D$ Green (La Jolla Institute for Allergy and Immunology, San Diego, CA, USA) and DW Nicholson (Merck Frosst, Canada). Bacterial expression plasmids for caspases -9 and -3 were kindly provided by Dr ES Alnemri (Kimmel Cancer Institute, Philadelphia, PA, USA). Mammalian expression plasmids for CD95 and Bax were kindly provided by Drs R Siegel (National Institute of Allergy and Infectious Diseases, National Institutes of Health, Bethesda, MD, USA) and S Korsmeyer (Howard Hughes Medical Institute, Harvard Medical School, Dana-Farber Cancer Institute, Boston, MA, USA). This work was partly funded by a European Union grant (QLG1-1999-00739 to GM Cohen).

\section{References}

1. Cohen GM (1997) Caspases: the executioners of apoptosis. Biochem. J.326:116

2. Earnshaw WC, Martins LM and Kaufmann SH (1999) Mammalian caspases: structure, activation, substrates, and functions during apoptosis. Annu. Rev. Biochem. 68: 383-424

3. Thornberry NA and Lazebnik Y (1998) Caspases: enemies within. Science 281: $1312-1316$

4. Bratton SB, MacFarlane M, Cain K and Cohen GM (2000) Protein complexes activate distinct caspase cascades in death receptor and stress-induced apoptosis. Exp. Cell Res. 256: 27-33 
5. Walczak H and Krammer PH (2000) The CD95 (APO-1/Fas) and the TRAIL (APO-2L) apoptosis systems. Exp. Cell Res. 256: 58-66

6. Bossy-Wetzel E, Newmeyer DD and Green DR (1998) Mitochondrial cytochrome c release in apoptosis occurs upstream of DEVD-specific caspase activation and independently of mitochondrial transmembrane depolarization. EMBO J.17: $37-49$

7. Liu X, Kim CN, Yang J, Jemmerson R and Wang X (1996) Induction of apoptotic program in cell-free extracts: requirement for dATP and cytochrome c. Cell 86: $147-157$

8. Harris MH and Thompson CB (2000) The role of the Bcl-2 family in the regulation of outer mitochondrial membrane permeability. Cell Death Differ. 7: 1182-1191

9. Cain K, Brown DG, Langlais C and Cohen GM (1999) Caspase activation involves the formation of the aposome, a large ( $\sim 700 \mathrm{kDa})$ caspase-activating complex. J. Biol. Chem. 274: 22686-22692

10. Cain K, Bratton SB, Langlais C, Walker G, Brown DG, Sun XM and Cohen GM (2000) Apaf-1 oligomerizes into biologically active $\sim 700 \mathrm{kDa}$ and inactive $\sim 1.4$ MDa apoptosome complexes. J. Biol. Chem. 275: 6067-6070

11. Li P, Nijhawan D, Budihardjo I, Srinivasula SM, Ahmad M, Alnemri ES and Wang X (1997) Cytochrome $c$ and dATP-dependent formation of Apaf-1/caspase-9 complex initiates an apoptotic protease cascade. Cell 91: 479-489

12. Saleh A, Srinivasula SM, Acharya S, Fishel R and Alnemri ES (1999) Cytochrome $c$ and dATP-mediated oligomerization of Apaf-1 is a prerequisite for procaspase-9 activation. J. Biol. Chem. 274: 17941-17945

13. Zou H, LiY, Liu X and Wang X (1999) An Apaf-1 cytochrome c multimeric complex is a functional apoptosome that activates procaspase-9. J. Biol. Chem. 274: $11549-11556$

14. Acehan D, Jiang X, Morgon DG, Heuser JE, Wang X and Akey CW (2002) Threedimensional structure of the apoptosome: implications for assembly, procaspase- 9 binding, and activation. Mol. Cell 9: 423-432

15. Rodriguez $\mathrm{J}$ and Lazebnik $Y$ (1999) Caspase-9 and APAF-1 form an active holoenzyme. Genes Dev. 13: 3179-3184

16. Bratton SB, Walker G, Srinivasula S, Sun X-M, Butterworth M, Alnemri ES and Cohen GM (2001) Recruitment, activation and retention of caspases- 9 and -3 by Apaf-1 apoptosome and associated XIAP complexes. EMBO J. 20: 998-2001

17. Stennicke HR, Deveraux QL, Humke EW, Reed JC, Dixit VM and Salvesen GS (1999) Caspase-9 can be activated without proteolytic processing. J. Biol. Chem. 274: 8359-8362

18. Srinivasula SM, Hegde R, Saleh A, Datta P, Shiozaki E, Chai J, Lee RA, Robbins PD, Fernandes-Alnemri T, Shi Y and Alnemri ES (2001) A conserved XIAPinteraction motif in caspase- 9 and Smac/DIABLO regulates caspase activity and apoptosis. Nature 410: 112-116

19. LiH, Zhu H, Xu CJ and Yuan J (1998) Cleavage of BID by caspase 8 mediates the mitochondrial damage in the Fas pathway of apoptosis. Cell 94: 491-501

20. Luo X, Budihardjo I, Zou H, Slaughter C and Wang X (1998) Bid, a Bcl2 interacting protein, mediates cytochrome $c$ release from mitochondria in response to activation of cell surface death receptors. Cell 94: $481-490$

21. Nicholson DW (1999) Caspase structure, proteolytic substrates, and function during apoptotic cell death. Cell Death Differ. 6: 1028-1042

22. Slee EA, Harte MT, Kluck RM, WolfBB, Casiano CA, Newmeyer DD, Wang HG, Reed JC, Nicholson DW, Alnemri ES, Green DR and Martin SJ (1999) Ordering the cytochrome $c$-initiated caspase cascade: hierarchical activation of caspases-2, -3, -6, -7, -8, and -10 in a caspase-9- dependent manner. J. Cell. Biol. 144: $281-292$

23. Clem RJ and Miller LK (1994) Control of programmed cell death by the baculovirus genes p35 and iap. Mol. Cell Biol. 14: 5212-5222

24. Deveraux QL and Reed JC (1999) IAP family proteins-suppressors of apoptosis. Genes Dev. 13: 239-252

25. Richter BW, Mir SS, Eiben LJ, Lewis J, Reffey SB, Frattini A, Tian L, Frank S, Youle RJ, Nelson DL, Notarangelo LD, Vezzoni P, Fearnhead HO and Duckett CS (2001) Molecular cloning of ILP-2, a novel member of the inhibitor of apoptosis protein family. Mol. Cell Biol. 21: 4292-4301

26. Silke $J$ and Vaux DL (2001) Two kinds of BIR-containing protein-inhibitors of apoptosis, or required for mitosis. J. Cell Sci. 114: 1821-1827

27. Chai J, Shiozaki E, Srinivasula SM, Wu Q, Datta P, Alnemri ES and Shi Y (2001) Structural basis of caspase-7 inhibition by XIAP. Cell 104: 769-780

28. Huang Y, Park YC, Rich RL, Segal D, Myszka DG and Wu H (2001) Structural basis of caspase inhibition by XIAP: differential roles of the linker versus the BIR domain. Cell 104: 781-790
29. Riedl SJ, Renatus M, Schwarzenbacher R, Zhou Q, Sun C, Fesik SW, Liddington RC and Salvesen GS (2001) Structural basis for the inhibition of caspase-3 by XIAP. Cell 104: 791-800.

30. Sun C, Cai M, Gunasekera AH, Meadows RP, Wang H, Chen J, Zhang H, Wu W, Xu N, Ng SC and Fesik SW (1999) NMR structure and mutagenesis of the inhibitor-of-apoptosis protein XIAP. Nature 401: 818-822

31. Sun C, Cai M, Meadows RP, Xu N, Gunasekera AH, Herrmann J, Wu JC and Fesik SW (2000) NMR structure and mutagenesis of the third Bir domain of the inhibitor of apoptosis protein XIAP. J. Biol. Chem. 275: 33777-33781

32. Suzuki Y, Nakabayashi Y, Nakata K, Reed JC and Takahashi R (2001) X-linked inhibitor of apoptosis protein (XIAP) inhibits caspase-3 and -7 in distinct modes. J. Biol. Chem. 276: 27058-27063

33. Takahashi R, Deveraux Q, Tamm I, Welsh K, Assa-Munt N, Salvesen GS and Reed JC (1998) A single BIR domain of XIAP sufficient for inhibiting caspases. J. Biol. Chem. 273: 7787-7790

34. Suzuki $Y$, Nakabayashi $Y$ and Takahashi R (2001) Ubiquitin-protein ligase activity of X-linked inhibitor of apoptosis protein promotes proteasomal degradation of caspase-3 and enhances its anti-apoptotic effect in Fas-induced cell death. Proc. Natl. Acad. Sci. USA 98: 8662-8667

35. Yang Y, Fang S, Jensen JP, Weissman AM and Ashwell JD (2000) Ubiquitin protein ligase activity of IAPs and their degradation in proteasomes in response to apoptotic stimuli. Science 288: 874-877

36. Du C, Fang M, Li Y, Li L and Wang X (2000) Smac, a mitochondrial protein that promotes cytochrome $\mathrm{c}$-dependent caspase activation by eliminating IAP inhibition. Cell 102: 33-42

37. Ekert PG, Silke J, Hawkins CJ, Verhagen AM and Vaux DL (2001) DIABLO promotes apoptosis by removing MIHA/XIAP from processed caspase 9 . J. Cell Biol. 152: 483-490

38. Srinivasula SM, Datta P, Fan XJ, Fernandes-Alnemri T, Huang Zand Alnemri ES (2000) Molecular Determinants of the Caspase-promoting Activity of Smac/ DIABLO andits Role in the Death Receptor Pathway. J. Biol. Chem. 275: 3615236157

39. Verhagen AM, Ekert PG, Pakusch M, Silke J, Connolly LM, Reid GE, Moritz RL, Simpson RJ and Vaux DL (2000) Identification of DIABLO, a mammalian protein that promotes apoptosis by binding to and antagonizing IAP proteins. Cell 102: $43-53$

40. Sun XM, Bratton SB, Butterworth M, MacFarlane M and Cohen GM (2002) Bcl-2 and $\mathrm{Bcl}-\mathrm{xL}$ inhibit $\mathrm{CD} 95$-mediated apoptosis by preventing mitochondrial release of Smac/DIABLO and subsequent inactivation of XIAP. J. Biol. Chem. 277: $11345-11351$

41. Birkey Reffey S, Wurthner JU, Parks WT, Roberts AB and Duckett CS (2001) Xlinked inhibitor of apoptosis protein functions as a cofactor in transforming growth factor- $\beta$ signaling. J. Biol. Chem. 28: 26542-26549

42. Hofer-Warbinek R, Schmid JA, Stehlik C, Binder BR, Lipp J and de Martin R (2000) Activation of NF-kappa B by XIAP, the X chromosome-linked inhibitor of apoptosis, in endothelial cells involves TAK1. J. Biol. Chem. 275:22064-22068

43. Sanna MG, Duckett CS, Richter BW, Thompson CB and Ulevitch RJ (1998) Selective activation of JNK1 is necessary for the anti-apoptotic activity of hILP. Proc. Natl. Acad. Sci. USA 95: 6015-6020

44. Yamaguchi K, Nagai S, Ninomiya-Tsuji J, Nishita M, Tamai K, Irie K, Ueno N, Nishida E, Shibuya H and Matsumoto K (1999) XIAP, a cellular member of the inhibitor of apoptosis protein family, links the receptors to TAB1-TAK1 in the BMP signaling pathway. EMBO J. 18: 179-187

45. Richter BWM and Duckett CS (2000) The IAP proteins: caspase inhibitors and beyond. www.stke.org/cgi/content/full/OC_sigtrans;2000/44/pe1:1-4

46. Silke J, Ekert PG, Day CL, Hawkins CJ, Baca M, Chew J, Pakusch M, Verhagen AM and Vaux DL (2001) Direct inhibition of caspase 3 is dispensable for the antiapoptotic activity of XIAP. EMBO J. 20: 3114-3123

47. Bratton SB, Walker G, Roberts DL, Cain K and Cohen GM (2001) Caspase-3 cleaves Apaf-1 into an approximately $30 \mathrm{kDa}$ fragment that associates with an inappropriately oligomerized and biologically inactive $\sim 1.4 \mathrm{MDa}$ apoptosome complex. Cell Death Differ. 8: 425-433

48. Deveraux QL, Leo E, Stennicke HR, Welsh K, Salvesen GS and Reed JC (1999) Cleavage of human inhibitor of apoptosis protein XIAP results in fragments with distinct specificities for caspases. EMBO. J. 18: 5242-5251

49. Johnson DE, Gastman BR, Wieckowski E, Wang GQ, Amoscato A, Delach SM and Rabinowich $\mathrm{H}(2000)$ Inhibitor of apoptosis protein hILP undergoes caspasemediated cleavage during T lymphocyte apoptosis. Cancer Res. 60: 1818-1823 
50. Liu Z, Sun C, Olejniczak ET, Meadows RP, Betz SF, Oost T, Herrmann J, Wu JC and Fesik SW (2000) Structural basis for binding of Smac/DIABLO to the XIAP BIR3 domain. Nature 408: 1004-1008

51. Wu G, Chai J, Suber TL, Wu JW, Du C, Wang X and Shi Y (2000) Structural basis of IAP recognition by Smac/DIABLO. Nature 408: 1008-1012

52. Chai J, Du C, Wu JW, Kyin S, Wang X and Shi $Y(2000)$ Structural and biochemical basis of apoptotic activation by Smac/DIABLO. Nature 406: $855-$ 862

53. Nicholson DW, Ali A, Thornberry NA, Vaillancourt JP, Ding CK, Gallant M, Gareau Y, Griffin PR, Labelle M, Lazebnik YA, Munday NA, Raju SM, Smulson ME, Yamin T-T, Yu VL and Miller DK (1995) Identification and inhibition of the ICE/CED-3 protease necessary for mammalian apoptosis. Nature 376: 37-43

54. Scaffidi C, Fulda S, Srinivasan A, Friesen C, Li F, Tomaselli KJ, Debatin KM Krammer PH and Peter ME (1998) Two CD95 (APO-1/Fas) signaling pathways. EMBO J. 17: 1675-1687

55. Deng $Y$, Lin $Y$ and $W u ~ X ~(2002)$ TRAIL-induced apoptosis requires Baxdependent mitochondrial release of Smac/DIABLO. Genes Dev. 16: 33-45

56. Zhang XD, Zhang XY, Gray CP, Nguyen T and Hersey P (2001) Tumor necrosis factor-related apoptosis-inducing ligand-induced apoptosis of human melanoma is regulated by smac/DIABLO release from mitochondria. Cancer Res. 61 : 7339-7348
57. Wang SL, Hawkins CJ, Yoo SJ, Muller HA and Hay BA (1999) The Drosophila caspase inhibitor DIAP1 is essential for cell survival and is negatively regulated by HID. Cell 98: $453-463$

58. Harlin H, Reffey SB, Duckett CS, Lindsten T and Thompson CB (2001) Characterization of XIAP-deficient mice. Mol. Cell Biol. 21: 3604-3608

59. Deveraux QL, Welsh K and Reed JC (2000) Purification and use of recombinant inhibitor of apoptosis proteins as caspase inhibitors. Methods Enzymol. 322: $154-161$

60. Roberts DL, Merrison W, MacFarlane M and Cohen GM (2001) The inhibitor of apoptosis protein-binding domain of Smac is not essential for its proapoptotic activity. J. Cell Biol. 153: 221-228

61. Duckett CS, LiF, Wang Y, Tomaselli KJ, Thompson CB and Armstrong RC (1998) Human IAP-like protein regulates programmed cell death downstream of $\mathrm{Bcl}-\mathrm{xL}$ and cytochrome c. Mol. Cell Biol. 18: 608-615 